\title{
Consolidation of Elastic Porous Media Saturated by Two IMMISCIBLE FLUIDS
}

\author{
By Kagan Tuncay ${ }^{1}$ and M. Yavuz Corapcioglu, ${ }^{2}$ Fellow, ASCE
}

\begin{abstract}
A theory is presented to simulate the consolidation of elastic porous media saturated by two immiscible Newtonian fiuids. The macroscopic equations, including mass and momentum balance equations and constitutive relations, are obtained by volume averaging the microscale equations. The theory is based on the small deformation assumption. In the microscale, the grains are assumed to be linearly elastic and the fluids are Newtonian. The bulk and shear moduli of the solid matrix are introduced to obtain the macroscopic constitutive equations. Momentum transfer terms are expressed in terms of intrinsic and relative permeabilities assuming the validity of Darcy's law. In one dimension, the governing equations reduce to two coupled diffusion equations in terms of the pore pressures of the fluid phases. An analytical solution is obtained for a column with a fixed impervious base and a free drainage surface. Results are presented for cases of practical interest, i.e., columns saturated by oil-water and air-water phases. Results indicate that the presence of a second fluid phase affects pore water pressure and total settlement.
\end{abstract}

\section{INTRODUCTION}

Many geological formations contain more than one fluid phase in their pores such as air, water, and oil. The interfacial tension between these fluids is nonzero and a fluid-fluid interface separates the phases within the pores. Although most earth materials are saturated by more than one fluid, consolidation of porous media saturated by multiphase fluids received limited attention from researchers. Almost all studies deal with soils containing air and water in their pores, i.e., unsaturated soils. In early studies, Terzaghi's (1923) effective stress principle was extended to unsaturated soils to incorporate the effect of air on pore water pressure. Among them, Bishop's (1959) principle is the most widely accepted one. Bishop introduced a material parameter to take the presence of air phase into consideration. Jennings and Burland (1962) showed that volume changes due to water wetting of the solid phase cannot be explained by Bishop's expression. For a detailed discussion of modified effective stress principle, the reader is referred to Bishop and Blight (1963), Kohgo et al. (1993), and Fredlund and Morgenstern (1977). However, we should note that these expressions were obtained based on practical considerations rather than theoretical ones.

To explain the behavioral alterations in volume change such as collapse of soil structure, various researchers proposed two stress states that are treated independently (Jennings and Burland 1962; Coleman 1962; Fredlund and Morgenstern 1976; Fredlund and Hasan 1979). In this approach, stress states are chosen as algebraic combinations of the stresses in the solid, air, and water phases. Then, constitutive relations for volume changes are expressed in terms of these stress states, e.g., as a function of the difference between total stress and air pressure, and the difference between air pressure and water pressure (Fredlund and Hasan 1979; Lloret and Alonso 1980). Fredlund and Hasan (1979) proposed a one-dimensional consolidation theory for unsaturated soils by considering volume changes and fluid flow due to variations in these two stress states. Their theory consists of two coupled diffusion equations

\footnotetext{
${ }^{1}$ Asst. Prof., Izmir Inst. of Technology, Facu. of Engrg., Gaziosmanpasa Bulvari, No. 16, Cankaya, Izmir, Turkey.

${ }^{2}$ Prof., Dept. of Civ. Engrg., Texas A\&M Univ., College Station, TX 77843-3136.

Note. Associate Editor: James T. Kirby. Discussion open until April 1, 1997. To extend the closing date one month, a written request must be filed with the ASCE Manager of Journals. The manuscript for this paper was submitted for review and possible publication on November 21, 1994. This paper is part of the Journal of Engineering Mechanics, Vol. 122, No. 11, November, 1996. CASCE, ISSN 0733-9399/96/0011$1077-1085 / \$ 4.00+\$ .50$ per page. Paper No. 9619.
}

in terms of air and water pore pressures. Fredlund and Hasan (1979) have expressed the flow of wetting (water) and nonwetting (air) phases by Darcy's and Fick's laws, respectively. Lloret and Alonso (1980) studied swelling and collapse behavior of unsaturated soils based on the same principles with the exception of Darcy's law expressed in terms of respective relative permeabilities for each phase. Narasimhan and Witherspoon $(1977,1978 \mathrm{a}, 1978 \mathrm{~b})$ proposed a numerical model for water flow in deforming unsaturated porous media based on Richards' equation and Bishop's effective stress principle. Corapcioglu and Bear (1983) have noted the contribution of the unsaturated zone above the water table to subsidence of a deformable phreatic aquifer. Geraminegad and Saxena (1986) developed a model to predict the coupled transient flow of heat, water, and air. They solved the resulting equations by the finite element method. Brutsaert and El-Kadi (1984) investigated the relative importance of partial saturation in ground-water flow by extending Biot's theory to unsaturated porous media.

Governing equations of compressible porous media can be obtained by using two different techniques. The first technique employs the mixture theory that treats the particulate volume fraction as a constitutive variable for multiphase media like compressible porous materials (Adkins 1963; Bowen 1982). The second technique volume averages the microscale governing equations to obtain macroscale ones (Slattery 1981). In the present study, we present a theory to simulate the consolidation of porous media saturated by two immiscible fluids. Mass and momentum balance equations as well as the constitutive relations are obtained by volume averaging the equations and relations expressed at the microscopic scale. The volume averaging technique has been employed after the development of the theorem for volume average of a gradient (Slattery 1967; Anderson and Jackson 1967; Marle 1967; Whitaker 1967). Although the volume averaging technique has been used extensively to formulate the flow problems in rigid porous media (Slattery 1967, 1968; Whitaker 1967), it has been recently applied to deformable media [e.g., refer to Bear et al. (1984)]. De la Cruz and Spanos (1985) made the first attempt to formulate the constitutive relations and balance equations of wave propagation in saturated porous media. In a subsequent paper, de la Cruz and Spanos (1989) extended their theory to include the thermodynamic considerations. Pride et al. (1992) obtained Biot's $(1941,1956)$ equations for saturated porous media by employing the volume averaging technique. The resulting constitutive relations of Pride et al. (1992) contained the same parameters as those of Biot and Willis (1957). As seen in this brief review, there is no theory available to obtain constitutive relations as well as mass and 
momentum balance equations at macroscale by volume averaging the corresponding microscopic ones. The resulting governing equations reduce to two coupled one-dimensional diffusion equations. These equations are similar to those obtained by Fredlund and Hasan (1979) when the external loads are not functions of time. Finally, we obtain analytical solutions for a porous column saturated by two immiscible fluids. Specific cases are studied for air-water and oil-water phases and the effects of a second phase on pore water pressure and consolidation are discussed.

\section{VOLUME AVERAGING THEOREMS}

Let $L, l$ and $r$ be the characteristic lengths of the macroscopic scale, averaging volume, and pore scale, respectively. The required condition for the volume averaging is (Slattery 1981)

$$
r<l<<L
$$

In the present study, we assume that this requirement is satisfied. We continue with the definitions used in volume averaging literature. Let $\mathbf{B}_{i}$ be a field quantity of phase $i$, then the volume average of $\mathbf{B}_{i}$ is defined as

$$
\left\langle\mathbf{B}_{i}\right\rangle=\frac{1}{V} \int_{R_{i}} \mathbf{B}_{i} d V
$$

where $V=$ averaging volume; and $R_{i}=$ region occupied by phase $i$. The intrinsic volume average of $\mathbf{B}_{i}$, i.e., the mean value of $\mathbf{B}_{i}$ in $R_{i}$, is given by

$$
\overline{\mathbf{B}}_{i}=\frac{1}{V_{i}} \int_{R_{i}} \mathbf{B}_{i} d V
$$

where $V_{i}=$ volume of phase $i$ in averaging volume. These two averages are related by

$$
\left\langle\mathbf{B}_{i}\right\rangle=\alpha_{i} \overline{\mathbf{B}}_{i}
$$

where $\alpha_{i}=$ volume fraction of phase $i$. Now we set the volume average theorem for a gradient and a time derivative (Slattery 1967,1981 )

$$
\begin{aligned}
\left\langle\boldsymbol{\nabla} \mathbf{B}_{i}\right\rangle=\boldsymbol{\nabla}\left\langle\mathbf{B}_{i}\right\rangle+\frac{1}{V} \int_{s_{i j}} \mathbf{B}_{i} \mathbf{n}_{i} d A & i \neq j, j=1, \ldots, N \\
\left\langle\frac{\partial \mathbf{B}_{i}}{\partial t}\right\rangle=\frac{\partial\left\langle\mathbf{B}_{i}\right\rangle}{\partial t}-\frac{1}{V} \int_{s_{i j}} \mathbf{B}_{i} \mathbf{u} \cdot \mathbf{n}_{i} d A & i \neq j, j=1, \ldots, N
\end{aligned}
$$

where $S_{i j}=$ interface between phase $i$ and phase $j ; \mathbf{n}_{i}=$ outward normal of $S_{i j} ; \mathbf{u} \cdot \mathbf{n}_{i}=$ speed of displacement of $S_{i j}$ into other phases; and $N=$ number of phases. The theorem of volume average of a divergence is stated as

$$
\left\langle\boldsymbol{\nabla} \cdot \mathbf{B}_{i}\right\rangle=\nabla \cdot\left\langle\mathbf{B}_{i}\right\rangle+\frac{1}{V} \int_{S_{i j}} \mathbf{B}_{i} \cdot \mathbf{n}_{i} d A \quad i \neq j, j=1, \ldots, N
$$

If $\left\langle\mathbf{B}_{i}\right\rangle$ is taken to be a constant, (5) and (6) take the following forms:

$$
\begin{gathered}
\nabla \alpha_{i}=-\frac{1}{V} \int_{S_{i j}} \mathbf{n}_{i} d A \quad i \neq j, j=1, \ldots, N \\
\frac{\partial \alpha_{i}}{\partial t}=+\frac{1}{V} \int_{S_{i j}} \mathbf{u} \cdot \mathbf{n}_{i} d A \quad i \neq j, j=1, \ldots, N
\end{gathered}
$$

\section{MICROSCOPIC CONSTITUTIVE RELATIONS, MASS AND MOMENTUM BALANCE EQUATIONS}

In the present study, the compressible porous medium consists of compressible solid grains, and two immiscible Newtonian fluids. We assume that there is no mass exchange between the phases. The solid phase is assumed to be initially at rest, linearly elastic, isotropic, and experiencing small deformations. Then the constitutive relations are given by

$$
\tau_{s}=K_{s} \boldsymbol{\nabla} \cdot \mathbf{u}_{s} \mathbf{I}+G_{s}\left[\boldsymbol{\nabla} \mathbf{u}_{s}+\left(\nabla \mathbf{u}_{s}\right)^{T}-\frac{2}{3} \boldsymbol{\nabla} \cdot \mathbf{u}_{s} \mathbf{I}\right]
$$

where $\mathbf{u}_{s}, \tau_{s}, K_{s}, G_{s}$, and $\mathbf{I}=$ displacement, incremental stress tensor, bulk modulus, shear modulus of solid phase, and unit tensor, respectively. The superscript $T$ denotes transpose of a tensor. We assume that both fluid phases are Newtonian with constitutive relations

$$
\boldsymbol{\tau}_{i}=-P_{i} \mathbf{I}+\mu_{i}\left[\nabla \mathbf{v}_{i}+\left(\boldsymbol{\nabla} \mathbf{v}_{i}\right)^{T}-\frac{2}{3} \boldsymbol{\nabla} \cdot \mathbf{v}_{i} \mathbf{I}\right] \quad i=1,2
$$

where $\mathbf{v}_{i}, \boldsymbol{\tau}_{i}, P_{i}$, and $\mu_{i}=$ velocity, incremental stress tensor, incremental pore fluid pressure, and shear viscosity of fluid phase $i$, respectively. In (11), the bulk viscosity of fluids is assumed to be negligible. The state equations of fluid phases are assumed to be in the form of

$$
\frac{1}{K_{i}} \frac{d P_{i}^{*}}{d t}=\frac{1}{\rho_{i}} \frac{d \rho_{i}}{d t} \quad i=1,2
$$

where $K_{i}, \rho_{i}$, and $P_{i}^{*}=$ bulk modulus, density, and pressure of phase $i$. The mass balance equations are expressed as

$$
\frac{1}{\rho_{i}} \frac{d \rho_{i}}{d t}=-\nabla \cdot \mathbf{v}_{i} \quad i=1,2
$$

By combining (12) and (13), we obtain

$$
\frac{1}{K_{i}} \frac{d P_{i}^{*}}{d t}=-\nabla \cdot \mathbf{v}_{i} \quad i=1,2
$$

The pressure increment $P_{i}$ can be written as

$$
-P_{i}=K_{i} \boldsymbol{\nabla} \cdot \mathbf{u}_{i} \quad i=1,2
$$

where $\mathbf{u}_{i}=$ displacement of fluid phase $i$ from reference position, i.e., incremental displacement. We continue with the momentum balance equation in terms of incremental stresses and velocities

$$
\boldsymbol{\nabla} \cdot \boldsymbol{\tau}_{j}=\rho_{j} \frac{d \mathbf{v}_{j}}{d t} \quad j=s, 1,2
$$

Since consolidation is a slow phenomenon, the inertial effects in (16) can be assumed to be negligible. We note that the body forces do not appear in (16) because equations are expressed in terms of incremental stresses. The boundary conditions at the solid-fluid interfaces are expressed as

$$
\mathbf{v}_{s}=\mathbf{v}_{i} \text { and } \boldsymbol{\tau}_{s} \cdot \mathbf{n}_{s}+\boldsymbol{\tau}_{i} \cdot \mathbf{n}_{i}=\mathbf{0} \text { on } S_{s i} \quad i=1,2
$$

where subscript (si) denotes interface between solid phase and fluid phase $i$; and $\mathbf{n}_{j}=$ unit outward vector normal to interface. The boundary conditions at the fluid-fluid interfaces are (Slattery 1981)

$$
\mathbf{v}_{1}=\mathbf{v}_{2} \text { and } \boldsymbol{\tau}_{1} \cdot \mathbf{n}_{1}+\boldsymbol{\tau}_{2} \cdot \mathbf{n}_{2}=\nabla_{\sigma} \gamma-2 H \gamma \mathbf{n} \text { on } S_{12}
$$

where $\nabla_{\sigma}, \gamma$, and $H=$ surface gradient operator, interfacial tension, and mean curvature of interface, respectively. The terms on the right side of (18) may be interpreted as the rate of momentum production per unit area of the phase interface. The first term on the right side incorporates the position dependency of surface tension upon the interface. Therefore, the surface gradient operator incorporates the spatial variation of the surface tension along the phase interface because of impurities and local temperature variations.

\section{MACROSCOPIC CONSTITUTIVE RELATIONS}

Our next step is to obtain macroscopic constitutive relations by averaging the microscopic relations. Volume averaging of (10) yields 


$$
\begin{aligned}
& \frac{1}{V} \int_{R_{s}} \tau_{s} d V=K_{s}\left[\nabla \cdot\left(\alpha_{s} \overline{\mathbf{u}_{s}}\right)+\frac{1}{V} \int_{S_{s i}} \mathbf{u}_{s} \cdot \mathbf{n}_{s} d A\right] \mathbf{I}+G_{s}\left\{\nabla\left(\alpha_{s} \overline{\mathbf{u}_{s}}\right)\right. \\
& \left.\quad+\left[\nabla\left(\alpha_{s} \overline{\mathbf{u}}_{s}\right)\right]^{T}-\frac{2}{3} \nabla \cdot\left(\alpha_{s} \overline{\mathbf{u}}_{s}\right) \mathbf{I}+\mathbf{K}_{s i}\right\} \quad i=1,2
\end{aligned}
$$

where

$$
\mathbf{K}_{s i}=\frac{1}{V} \int_{S_{s i}}\left(\mathbf{u}_{s} \mathbf{n}_{s}+\mathbf{n}_{s} \mathbf{u}_{s}-\frac{\mathbf{2}}{\mathbf{3}} \mathbf{u}_{s} \cdot \mathbf{n}_{s} \mathbf{I}\right) d A \quad i=1,2
$$

is second-order tensor with zero trace. Since there is no mass exchange between the phases, the velocity of the interface is equal to the velocity of the points at the interface. Hence, by employing (9), the integral in (19) can be expressed as

$$
\frac{1}{V} \int_{S_{s i}} \mathbf{u}_{s} \cdot \mathbf{n}_{s} d A=\left(\alpha_{s}-\alpha_{s}^{0}\right)=\Delta \alpha_{s} \quad i=1,2
$$

where superscript $(0)$ refers to reference configuration. Since the displacements are assumed to be small, by definition

$$
\mathbf{u}_{j} \cdot \nabla \alpha_{j} \approx 0 \quad j=s, 1,2
$$

Then volume averaged constitutive relations for the solid phase (19) can be expressed as

$$
\begin{gathered}
\alpha_{s} \overline{\boldsymbol{\tau}}_{s}=K_{s}\left(\alpha_{s} \nabla \cdot \overline{\mathbf{u}}_{s}+\Delta \boldsymbol{\alpha}_{s}\right) \mathbf{I}+G_{s}\left[\alpha_{s} \nabla \overline{\mathbf{u}_{s}}+\alpha_{s}\left(\nabla \overline{\mathbf{u}_{s}}\right)^{T}\right. \\
\left.-\frac{2}{3} \alpha_{s} \nabla \cdot \overline{\mathbf{u}}_{s} \mathbf{I}+\mathbf{K}_{s i}\right] \quad i=1,2
\end{gathered}
$$

where $\overline{\tau_{s}}=$ intrinsic averaged incremental stress of solid phase. Similarly, the volume averaged constitutive relations for fluid phases are

$$
\begin{gathered}
\alpha_{i} \overline{\boldsymbol{\tau}}_{i}=K_{i}\left(\alpha_{l} \boldsymbol{\nabla} \cdot \overline{\mathbf{u}}_{i}+\Delta \alpha_{i}\right) \mathbf{I}+\mu_{i}\left[\alpha_{i} \nabla \overline{\mathbf{v}}_{i}+\alpha_{i}\left(\nabla \bar{\nabla} \overline{\mathbf{v}}_{l}\right)^{T}\right. \\
\left.-\frac{2}{3} \alpha_{i} \boldsymbol{\nabla} \cdot \overline{\mathbf{v}}_{i} \mathbf{I}+\mathbf{J}_{i s}+\mathbf{J}_{i k}\right] \quad i \neq k, i=1,2
\end{gathered}
$$

where

$$
\mathbf{J}_{i j}=\frac{1}{V} \int_{S_{i j}}\left(\mathbf{v}_{i} \mathbf{n}_{i}+\mathbf{n}_{i} \mathbf{v}_{i}-\frac{2}{3} \mathbf{v}_{i} \cdot \mathbf{n}_{i} \mathbf{I}\right) d A
$$

Under the small deformations assumption, the interfaces of the phases are not allowed to experience large deformations. Then by using (20) and (25), and assuming $\partial \mathbf{u}_{i} / \partial t \gg \mathbf{v}_{i} \cdot \nabla \mathbf{u}_{i}$, we can write

$$
\mathbf{J}_{i j}=\frac{\partial \mathbf{K}_{i j}}{\partial t}
$$

Furthermore, employing the no-slip conditions (17) we can write

$$
\mathbf{K}_{j k}=-\mathbf{K}_{k j} \quad \text { and } \quad \mathbf{J}_{j k}=-\mathbf{J}_{k j}
$$

$\mathbf{K}_{i j}$ and $\mathbf{J}_{i j}$ couple the shear deformation of the phases. However, we must note that physical meanings of the shear modulus of the solid phase and viscosity of the fluid phases are totally different. In almost all studies associated with the deformation of the solid matrix, these coupling terms are neglected assuming that all shear resistance is provided by the matrix only.

The microscopic boundary condition at the fluid-fluid interface [refer to (18)] shows that there is a jump in the stresses of the immiscible fluids because of the presence of interfacial tension and curvature of the interface. Assuming smooth pressure variations within the averaging volume, we can write

$$
\overline{P_{1}^{*}}-\overline{P_{2}^{*}}=P_{\text {cap }}\left(S_{1}\right)
$$

where $\overline{P_{1}^{*}}$ and $\overline{P_{2}^{*}}=$ intrinsic averaged pressures. $P_{\text {cap }}$, also known as capillary pressure, is assumed to be a function of $S_{1}$ (saturation of the nonwetting phase) only. From now on fluid phase 1 will be considered as the nonwetting phase and fluid phase 2 will be considered as the wetting phase. $S_{i}$ is related to the volume fractions by

$$
S_{i}=\frac{\alpha_{i}}{1-\alpha_{s}} \quad i=1,2
$$

Then

$$
S_{1}+S_{2}=1
$$

Since the fluid pressures we are working with are incremental pressures, as a first-order approximation, we can write

$$
\overline{P_{1}}-\overline{P_{2}}=\frac{d P_{\text {cap }}}{d S_{1}} \Delta S_{1}
$$

provided that change in saturation, $\Delta S_{1}$, is small.

Deformation of a porous medium can be investigated by independently considering the volume change behavior (nonzero trace) and shear deformation behavior (zero trace). In the following, we first consider the part of constitutive relations associated with the volume changes. After examining the shear deformations, we combine these two to finalize the macroscopic constitutive relations.

To explore the constitutive relations associated with the volume changes, we start by introducing $\bar{P}_{j}$

$$
-\alpha_{j} \bar{P}_{j}=\frac{1}{3} \operatorname{tr}\left(\alpha_{j} \bar{\tau}_{j}\right)=K_{j}\left(\alpha_{j} \nabla \cdot \overline{\mathbf{u}}_{j}+\Delta \alpha_{j}\right) \quad j=s, 1,2
$$

Eq. (32) does not contain any rotational deformations. For an elastic porous medium saturated by a single fluid, Pride et al. (1992) showed that

$$
\boldsymbol{\nabla} \cdot \overline{\mathbf{u}_{s}}=-\alpha_{s} \frac{\left(\overline{P_{s}}-\bar{P}_{f}\right)}{K_{\mathrm{fr}}}-\frac{\bar{P}_{f}}{K_{s}}
$$

where $K_{\mathrm{fr}}$ is defined as "frame" or "drained" bulk modulus. We assume that in the case of two fluids, $\widetilde{P}_{f}$ is given by

$$
\overline{P_{f}}=S_{1} \overline{P_{1}}+\left(1-S_{1}\right) \overline{P_{2}}
$$

Then, we can rewrite (33) as

$$
\boldsymbol{\nabla} \cdot \overline{\mathbf{u}}_{s}=-\alpha_{s} \frac{\left[\overline{P_{s}}-S_{1} \overline{P_{1}}-\left(1-S_{1}\right) \overline{P_{2}}\right]}{K_{\mathrm{fr}}}-\frac{\left[S_{1} \overline{P_{1}}+\left(1-S_{1}\right) \overline{P_{2}}\right]}{K_{s}}
$$

Note that from (29) and (30) we can write

$$
\begin{gathered}
\Delta \alpha_{1}=\Delta\left[S_{1}\left(1-\alpha_{s}\right)\right]=S_{1}\left(1-\alpha_{s}\right)-S_{1}^{0}\left(1-\alpha_{s}^{0}\right)=\left(1-\alpha_{s}^{0}\right) \Delta S_{1} \\
-S_{1}^{0} \Delta \alpha_{s}-\Delta \alpha_{s} \Delta S_{1} \approx\left(1-\alpha_{s}\right) \Delta S_{1}-S_{1} \Delta \alpha_{s}
\end{gathered}
$$

and

$$
\begin{aligned}
& \Delta \alpha_{2}=\Delta\left[\left(1-S_{1}\right)\left(1-\alpha_{s}\right)\right]=\left(1-S_{1}\right)\left(1-\alpha_{s}\right)-\left(1-S_{1}^{0}\right)\left(1-\alpha_{s}^{0}\right) \\
& \quad=-\left(1-\alpha_{s}^{0}\right) \Delta S_{1}-\left(1-S_{1}^{0}\right) \Delta \alpha_{s}+\Delta \alpha_{s} \Delta S_{1} \\
& \quad \approx-\left(1-\alpha_{s}\right) \Delta S_{1}-\left(1-S_{1}\right) \Delta \alpha_{s}
\end{aligned}
$$

Since we have already assumed that $\Delta S_{1}$ and $\Delta \alpha_{s}$ are small, the product of these terms can be neglected. Eqs. (31), (32), and (35) can be rewritten as

$$
\Delta \alpha_{s}=-\frac{A_{1}}{K_{s}^{2}}\left[S_{1} \overline{P_{1}}+\left(1-S_{1}\right) \overline{P_{2}}+K_{s} \boldsymbol{\nabla} \cdot \overline{\mathbf{u}_{s}}\right]
$$




$$
\begin{gathered}
\Delta S_{1}=\frac{\overline{P_{1}}-\overline{P_{2}}}{d P_{\text {cap }} / d S_{1}} \\
-\alpha_{s} \overline{P_{s}}=-\frac{A_{1}}{K_{s}}\left[S_{1} \overline{P_{1}}+\left(1-S_{1}\right) \overline{P_{2}}\right]+K_{f s} \nabla \cdot \overline{\mathbf{u}_{s}} \\
\nabla \cdot \overline{\mathbf{u}_{1}}=-d_{11} \nabla \cdot \overline{\mathbf{u}_{s}}-d_{12} \overline{P_{1}}+d_{13} \overline{P_{2}} \\
\nabla \cdot \overline{\mathbf{u}_{2}}=-d_{21} \nabla \cdot \overline{\mathbf{u}_{s}}+d_{22} \overline{P_{1}}-d_{23} \overline{P_{2}}
\end{gathered}
$$

where

$$
\begin{gathered}
d_{11}=\frac{A_{1}}{K_{s}\left(1-\alpha_{s}\right)} \\
d_{12}=\frac{K_{s}^{2}\left(1-\alpha_{s}\right)\left[K_{1}+A_{2} /\left(1-S_{1}\right)\right]+d P_{\mathrm{cap}} / d S_{1} S_{1}^{2} K_{1} A_{1}}{K_{1} K_{s}^{2} d P_{\mathrm{cap}} / d S_{1} S_{1}\left(1-\alpha_{s}\right)} \\
d_{13}=\frac{K_{s}^{2}\left(1-\alpha_{s}\right)-A_{2} A_{1}}{K_{s}^{2} d P_{\mathrm{cap}} / d S_{1} S_{1}\left(1-\alpha_{s}\right)} \\
d_{21}=\frac{A_{1}}{K_{s}\left(1-\alpha_{s}\right)} \\
d_{22}=\frac{K_{s}^{2}\left(1-\alpha_{s}\right)-A_{2} A_{1}}{K_{s}^{2} d P_{\mathrm{cap}} / d S_{1}\left(1-S_{1}\right)\left(1-\alpha_{s}\right)} \\
d_{23}=\frac{K_{s}^{2}\left(1-\alpha_{s}\right)\left(K_{2}+A_{2} / S_{1}\right)+d P_{\mathrm{cap}} / d S_{1}\left(1-S_{1}\right)^{2} K_{2} A_{1}}{K_{2} K_{s}^{2} d P_{\mathrm{cap}} / d S_{1}\left(1-S_{1}\right)\left(1-\alpha_{s}\right)} \\
A_{1}=\alpha_{s} K_{s}-K_{\mathrm{fr}} \quad \text { and } A_{2}=\frac{d P_{\text {cap }}}{d S_{1}} S_{1}\left(1-S_{1}\right)
\end{gathered}
$$

These constitutive relations simplify significantly if the solid grains are assumed to be incompressible, i.e., $K_{s} \rightarrow \infty$. We obtain an expression for $\Delta \alpha_{s}$ by substituting (35) in (32) where $j=s$

$$
\Delta \alpha_{s}=\left(\frac{\alpha_{s}^{2}}{K_{\mathrm{fr}}}-\frac{\alpha_{s}}{K_{s}}\right)\left[\overline{P_{s}}-S_{1} \overline{P_{1}}-\left(1-S_{1}\right) \overline{P_{2}}\right]
$$

We also note that $-\Delta \alpha_{s}$ is equal to the porosity change.

Our next step is the evaluation of the solid matrix's shear modulus, $G_{\mathrm{fr}}$. As noted earlier, we assume that all shear resistance of the porous material is provided by the solid matrix. This assumption uncouples the shear deformation of the phases, i.e., $\mathbf{K}_{i j}=\mathbf{J}_{i j}=\mathbf{0}$. If an external shear stress, $\boldsymbol{\tau}_{e}$, is applied to the material, then by employing (23) we can write

$$
\begin{aligned}
\tau_{e} & =\alpha_{s} \overline{\tau_{s}^{D}}+\alpha_{1} \overline{\tau_{1}^{D}}+\alpha_{2} \overline{\tau_{2}^{D}} \approx \alpha_{s} \overline{\tau_{s}} \\
& =G_{\mathrm{fr}}\left[\nabla \overline{\mathbf{u}_{s}}+\left(\nabla \overline{\mathbf{u}_{s}}\right)^{T}-\frac{2}{3} \nabla \cdot \overline{\mathbf{u}_{s}} \mathbf{I}\right]
\end{aligned}
$$

where $\overline{\tau_{j}^{D}}=$ deviatoric stress of phase $j$. In other words, the fluids are viscous but the mechanical shear response of the porous medium is provided by the solid matrix only. The viscosities of the fluids will be taken into account later when we discuss the macroscopic momentum balance equations since they contribute to the energy losses in the system. Then, we can finalize the constitutive relations as

- For the solid matrix

$$
\begin{aligned}
& \alpha_{s} \overline{\bar{\tau}_{s}}=\left\{K_{\mathrm{fr}} \boldsymbol{\nabla} \cdot \overline{\mathbf{u}_{s}}-\frac{A_{1}}{K_{s}}\left[S_{1} \overline{P_{1}}+\left(1-S_{1}\right) \overline{P_{2}}\right]\right\} \mathbf{I} \\
& +G_{\mathrm{fr}}\left[\nabla \overline{\mathbf{u}_{s}}+\left(\nabla \overline{\mathbf{u}_{s}}\right)^{r}-\frac{2}{3} \nabla \cdot \overline{\mathbf{u}_{s}} \mathbf{I}\right]
\end{aligned}
$$

- For the nonwetting fluid

$$
\alpha_{1} \overline{\tau_{1}}=-\alpha_{1} \overline{P_{1}} \mathbf{I}
$$

- For the wetting fluid

$$
\alpha_{2} \overline{T_{2}}=-\alpha_{2} \overline{P_{2}} \mathbf{I}
$$

Eqs. (52) $-(54)$ and (38) $-(42)$ constitute the complete set of constitutive relations of the porous media. These equations can be reduced to a case with a single fluid phase by setting $d P_{\text {cap }} / d S_{1} \rightarrow 0$ (i.e., $\Delta S_{1} \rightarrow 0$ ) and $S_{1}=0$. In this case, the definitions of the material constants are identical to those given by Biot and Willis (1957). We should note that in the present study we are considering a linearly elastic medium experiencing deformations. In some practical situations, the material parameters are highly nonlinear functions of state variables. The sum of volume-averaged stresses in all phases (52)-(54) yields the so-called "total stress" definition in soil mechanics. Hence

$$
\begin{aligned}
\left\langle\boldsymbol{\tau}_{t}\right\rangle & =K_{\mathrm{fr}} \boldsymbol{\nabla} \cdot \overline{\mathbf{u}_{s}} \mathbf{I}+G_{\mathrm{fr}}\left[\boldsymbol{\nabla} \overline{\mathbf{u}_{s}}+\left(\boldsymbol{\nabla} \overline{\mathbf{u}_{s}}\right)^{T}-\frac{2}{3} \boldsymbol{\nabla} \cdot \overline{\mathbf{u}_{s}} \mathbf{I}\right] \\
- & \left(1-\frac{K_{\mathrm{fr}}}{K_{s}}\right)\left[S_{1} \overline{P_{1}}+\left(1-S_{1}\right) \overline{P_{2}}\right] \mathbf{I}
\end{aligned}
$$

For a porous medium saturated by a single phase fluid, the effective stress $\left\langle\boldsymbol{\tau}_{\text {eff }}\right\rangle$ is defined as

$$
\left\langle\boldsymbol{\tau}_{i}\right\rangle=\left\langle\boldsymbol{\tau}_{\mathrm{eff}}\right\rangle-\beta \bar{P} \mathbf{I}
$$

where $\beta=a$ constant depending on elastic properties of medium. $\beta=1$ corresponds to the effective stress principle introduced by Terzaghi (1923). Later, Biot and Willis (1957), Skempton (1960), and Nur and Byerlee (1971) proposed

$$
\beta=1-\frac{K_{\mathrm{fr}}}{K_{s}}
$$

for a linearly elastic solid matrix. Usually the bulk modulus of the solid grains, $K_{s}$, is much greater than the bulk modulus of the frame, $K_{\mathrm{fr}}$. Then $\beta$ can be taken as unity for most cases. The lower limit of $\beta$ is $\left(1-\alpha_{s}\right)$ since $K_{\mathrm{fr}}$ cannot be greater than $\alpha_{s} K_{s}$. Similar effective stress principles were proposed for porous media saturated by two immiscible fluids. Bishop (1959) proposed

$$
\left\langle\boldsymbol{\tau}_{t}\right\rangle=\left\langle\boldsymbol{\tau}_{\text {eff }}\right\rangle-\overline{P_{1}} \mathbf{I}+\chi\left(\overline{P_{1}}-\overline{P_{2}}\right) \mathbf{I}
$$

where $\chi=$ material parameter. Bishop and Blight (1963) investigated the relationship between $\chi$ and $S_{2}$, and observed almost a linear variation.

The idea behind the effective stress principle is to find the intergranular stress that causes the deformation of the solid matrix. If effective stress on the solid matrix is known, the problem reduces to finding the response of the solid matrix by using the "drained" matrix parameters. Hence, (55) can be rewritten as

$$
\left\langle\boldsymbol{\tau}_{t}\right\rangle=\left\langle\boldsymbol{\tau}_{\text {eff }}\right\rangle-\left(1-\frac{K_{\mathrm{fr}}}{K_{s}}\right)\left[S_{1} \overline{P_{1}}+\left(1-S_{1}\right) \overline{P_{2}}\right] \mathbf{I}
$$

Eq. (59) is a combination of (56), (57), and (58) for $\chi=S_{2}$ and it can be regarded as a generalized effective stress principle for linearly elastic porous media saturated by two immiscible fluids.

\section{MACROSCOPIC EQUILIBRIUM EQUATIONS}

Using the averaging theorems, the volume average of macroscopic momentum balance (16) is obtained as

$$
\boldsymbol{\nabla} \cdot\left\langle\boldsymbol{\tau}_{j}\right\rangle+\frac{1}{V} \int_{S_{j i}} \boldsymbol{\tau}_{j} \cdot \mathbf{n}_{j} d A=\mathbf{0} \quad i=s, 1,2
$$


The second term on the left side represents momentum transfer between phases. Boundary conditions at the solid-fluid interfaces [refer to (17)] imply that

$$
\begin{aligned}
& \frac{1}{V} \int_{S_{s 1}} \boldsymbol{\tau}_{s} \cdot \mathbf{n}_{s} d A=-\frac{1}{V} \int_{S_{1 s}} \boldsymbol{\tau}_{1} \cdot \mathbf{n}_{1} d A \\
& \frac{1}{V} \int_{S_{s 2}} \boldsymbol{\tau}_{s} \cdot \mathbf{n}_{s} d A=-\frac{1}{V} \int_{S_{2 s}} \boldsymbol{\tau}_{2} \cdot \mathbf{n}_{2} d A
\end{aligned}
$$

Assuming that Darcy's law is valid, we can write the following by substituting (59) in (60):

$$
\begin{aligned}
& -\frac{1}{V} \int_{S_{1},} \tau_{1} \cdot \mathbf{n}_{1} d A=-\frac{\left(1-\alpha_{s}\right)^{2} S_{1}^{2} \mu_{1}}{K k_{r 1}}\left(\overline{\mathbf{v}_{1}}-\overline{\mathbf{v}}_{s}\right)=\left(1-\alpha_{s}\right) S_{1} \nabla \overline{P_{1}} \\
& -\frac{1}{V} \int_{S_{2 s}} \tau_{2} \cdot \mathbf{n}_{2} d A=-\frac{\left(1-\alpha_{s}\right)^{2}\left(1-S_{1}\right)^{2} \mu_{2}}{K k_{r 2}}\left(\overline{\mathbf{v}_{2}}-\overline{\mathbf{v}_{s}}\right) \\
& =\left(1-\alpha_{s}\right)\left(1-S_{1}\right) \nabla \overline{P_{2}}
\end{aligned}
$$

where $K=$ intrinsic permeability of medium; and $k_{r l}=$ relative permeability of phase $i$. Because of the boundary condition at the fluid-fluid interfaces [refer to (18)] we can write

$$
\frac{1}{V} \int_{S_{12}} \tau_{1} \cdot \mathbf{n}_{1} d A \neq-\frac{1}{V} \int_{s_{21}} \tau_{2} \cdot \mathbf{n}_{2} d A
$$

These terms result in the cross permeabilities known as the Yuster effect in the literature (Yuster 1953; Scott and Rose 1953). The Yuster effect can be neglected for practical purposes (Bear 1972). Summing the momentum balance of the solid and fluid phases [refer to (60)], we obtain the macroscopic equilibrium equations of the porous medium

$$
\boldsymbol{\nabla} \cdot\left\langle\boldsymbol{r}_{t}\right\rangle=\mathbf{0}
$$

\section{FINAL SET OF EQUATIONS}

Based on the set of macroscopic constitutive relations and momentum balance equations, equations governing the consolidation of a porous medium saturated by two immiscible fluids can be stated. By applying the divergence operator to (62) we have

$$
\begin{gathered}
\left(1-\alpha_{s}\right) S_{1} \nabla \cdot\left(\overline{\mathbf{v}_{1}}-\overline{\mathbf{v}}_{s}\right)=\nabla \cdot\left(-\frac{K k_{r 1}}{\mu_{1}} \nabla \overline{P_{1}}\right) \\
\left(1-\alpha_{s}\right)\left(1-S_{1}\right) \nabla \cdot\left(\overline{\mathbf{v}_{2}}-\overline{\mathbf{v}_{s}}\right)=\nabla \cdot\left(-\frac{K k_{r 2}}{\mu_{2}} \nabla \overline{P_{2}}\right)
\end{gathered}
$$

In writing (65) and (66), we assume that gradients of volume fractions are small. By employing the constitutive relations given by (41) and (42), we eliminate the fluid velocities from (65) and (66) to obtain

$$
\begin{aligned}
& -S_{1}\left(1-\alpha_{s}\right) d_{12} \frac{\partial \overline{P_{1}}}{\partial t}+S_{1}\left(1-\alpha_{s}\right) d_{13} \frac{\partial \overline{P_{2}}}{\partial t} \\
& =\nabla \cdot\left(-\frac{K k_{r 1}}{\mu_{1}} \nabla \overline{P_{1}}\right)+\left(1-\alpha_{s}\right)\left(1+d_{11}\right) S_{1} \nabla \cdot \overline{v_{s}} \\
& \left(1-S_{1}\right)\left(1-\alpha_{s}\right) d_{22} \frac{\partial \bar{P}_{1}}{\partial t}-\left(1-S_{1}\right)\left(1-\alpha_{s}\right) d_{23} \frac{\partial \overline{P_{2}}}{\partial t} \\
& =\nabla \cdot\left(-\frac{K k_{r 2}}{\mu_{2}} \nabla \overline{P_{2}}\right)+\left(1-\alpha_{s}\right)\left(1+d_{21}\right)\left(1-S_{1}\right) \nabla \cdot \overline{\mathbf{v}}
\end{aligned}
$$

Then, substitution of (55) in (64) yields

$$
\begin{aligned}
\boldsymbol{\nabla} & {\left[\left(K_{\mathrm{fr}}+\frac{1}{3} G_{\mathrm{fr}}\right) \nabla \cdot \overline{\mathbf{u}_{s}}\right]+\nabla \cdot\left(G_{\mathrm{fr}} \nabla \overline{\mathbf{u}_{s}}\right) } \\
& -\boldsymbol{\nabla}\left\{\left(1-\frac{K_{\mathrm{fr}}}{K_{s}}\right)\left[S_{1} \overline{P_{1}}+\left(1-S_{1}\right) \overline{P_{2}}\right]\right\}=\mathbf{0}
\end{aligned}
$$

Eqs. (67), (68), and (69) constitute the final set of equations with the unknowns $\overline{P_{1}}, \overline{P_{2}}$, and $\overline{\mathbf{u}_{s}}$.

\section{ONE-DIMENSIONAL CONSOLIDATION PROBLEM}

In general, (67)-(69) have five unknowns: $\overline{P_{1}}, \overline{P_{2}}$, and three components of the solid matrix displacement, $\overline{\mathbf{u}}_{s}$. For a onedimensional consolidation of a column, (67)-(69) reduce to

$$
\begin{aligned}
& -S_{1}\left(1-\alpha_{s}\right) d_{12} \frac{\partial \overline{P_{1}}}{\partial t}+S_{1}\left(1-\alpha_{s}\right) d_{13} \frac{\partial \overline{P_{2}}}{\partial t} \\
& =\frac{\partial}{\partial z}\left(-\frac{K k_{r 1}}{\mu_{1}} \frac{\partial \overline{P_{1}}}{\partial z}\right)+\left(1-\alpha_{s}\right)\left(1+d_{11}\right) S_{1} \frac{\partial^{2} \overline{w_{s}}}{\partial z \partial t} \\
& \left(1-S_{1}\right)\left(1-\alpha_{s}\right) d_{22} \frac{\partial \overline{P_{1}}}{\partial t}-\left(1-S_{1}\right)\left(1-\alpha_{s}\right) d_{23} \frac{\partial \overline{P_{2}}}{\partial t} \\
& =\frac{\partial}{\partial z}\left(-\frac{K k_{r 2}}{\mu_{2}} \frac{\partial \overline{P_{2}}}{\partial z}\right)+\left(1-\alpha_{s}\right)\left(1+d_{21}\right)\left(1-S_{1}\right) \frac{\partial^{2} \overline{w_{s}}}{\partial z \partial t} \\
& \frac{\partial}{\partial z}\left[\left(K_{\mathrm{fr}}+\frac{4}{3} G_{\mathrm{fr}}\right) \frac{\partial \bar{w}_{s}}{\partial z}\right] \\
& \quad-\frac{\partial}{\partial z}\left\{\left(1-\frac{K_{\mathrm{fr}}}{K_{s}}\right)\left[S_{1} \overline{P_{1}}+\left(1-S_{1}\right) \overline{P_{2}}\right]\right\}=0
\end{aligned}
$$

where $\overline{w_{s}}=$ vertical displacement of solid matrix. Eqs. (70)(72) can be solved for a two-phase saturated column with fixed impervious base and free drainage surface. We take the $z$-axis positive downward. The thickness of the column is $h_{0}$. We assume that material parameters are constant and uniform along the column. Since the total stress is constant at any depth, (55) can be rewritten as

$$
-P_{0}=\left(K_{\mathrm{fr}}+\frac{4}{3} G_{\mathrm{fr}}\right) \frac{\partial \overline{w_{s}}}{\partial z}-\left(1-\frac{K_{\mathrm{fr}}}{K_{s}}\right)\left[S_{1} \overline{P_{1}}+\left(1-S_{1}\right) \overline{P_{2}}\right]
$$

where $P_{0}=$ applied stress at top, $z=0$. Substitution of (73) in (70) and (71) yields

$$
\begin{aligned}
& a_{11} \frac{\partial \overline{P_{1}}}{\partial t}+a_{12} \frac{\partial \overline{P_{2}}}{\partial t}=e_{1} \frac{\partial^{2} \overline{P_{1}}}{\partial z^{2}} \\
& a_{21} \frac{\partial \overline{P_{1}}}{\partial t}+a_{22} \frac{\partial \overline{P_{2}}}{\partial t}=e_{2} \frac{\partial^{2} \overline{P_{2}}}{\partial z^{2}}
\end{aligned}
$$

where

$$
\begin{aligned}
a_{11} & =S_{1}\left(1-\alpha_{s}\right) d_{12}+\frac{\left(1-\alpha_{s}\right)\left(1+d_{11}\right)\left(1-K_{\mathrm{fr}} / K_{s}\right) S_{1}^{2}}{K_{\mathrm{fr}}+4 G_{\mathrm{fr}} / 3} \\
a_{12} & =-S_{1}\left(1-\alpha_{s}\right) d_{13}+\frac{\left(1-\alpha_{s}\right)\left(1+d_{11}\right)\left(1-K_{\mathrm{fr}} / K_{s}\right)\left(1-S_{1}\right) S_{1}}{K_{\mathrm{fr}}+4 G_{\mathrm{fr}} / 3} \\
a_{21} & =-\left(1-S_{1}\right)\left(1-\alpha_{s}\right) d_{22} \\
& +\frac{\left(1-\alpha_{s}\right)\left(1+d_{21}\right)\left(1-K_{\mathrm{fr}} / K_{s}\right)\left(1-S_{1}\right) S_{1}}{K_{\mathrm{fr}}+4 G_{\mathrm{fr}} / 3} \\
a_{22} & =\left(1-S_{1}\right)\left(1-\alpha_{s}\right) d_{23}+\frac{\left(1-\alpha_{s}\right)\left(1+d_{21}\right)\left(1-K_{\mathrm{fr}} / K_{s}\right)\left(1-S_{1}\right)^{2}}{K_{\mathrm{fr}}+4 G_{\mathrm{fr}} / 3}
\end{aligned}
$$

and

$$
\begin{aligned}
& e_{1}=\frac{K k_{r 1}}{\mu_{1}} \\
& e_{2}=\frac{K k_{r 2}}{\mu_{2}}
\end{aligned}
$$


Eqs. (74) and (75) are the governing equations of one-dimensional consolidation when external loads are not functions of time. These equations are similar to those obtained by Fredlund and Hasan (1979) for unsaturated soils. However, the constants are not similar because of different approaches used in the derivations. The boundary conditions are

$$
\begin{array}{cccc}
\overline{P_{1}} & =\overline{P_{2}}=0 \quad \text { at } & z=0 \\
\frac{\partial \overline{P_{1}}}{\partial z}=\frac{\partial \overline{P_{2}}}{\partial z}=0 & \text { at } & z=h_{0}
\end{array}
$$

Initial changes in volume fractions are zero, i.e., $\Delta \alpha_{s}=\Delta S_{1}=$ 0 at $t=0$. Then, from (38) and (39) we can write

$$
S_{1} \overline{P_{1}}+\left(1-S_{1}\right) \overline{P_{2}}+K_{s} \frac{\partial \overline{W_{s}}}{\partial z}=0 \text { at } t=0
$$

and

$$
\frac{\overline{P_{1}}-\overline{P_{2}}}{d P_{\text {cap }} / d S_{1}}=0 \text { at } t=0
$$

Substitution of (73) and (85) in (84) gives the initial condition

$$
\overline{P_{1}}=\overline{P_{2}}=P_{0} \frac{K_{s}}{K_{\mathrm{fr}}+4 G_{\mathrm{fr}} / 3+K_{s}\left(1-K_{\mathrm{fr}} / K_{s}\right)}=P_{0}^{*} \quad \text { at } \quad t=0
$$

The general solutions of (74) and (75) can be expressed as Fourier series

$$
\begin{aligned}
& \overline{P_{1}}(z, t)=\sum_{n=0}^{\infty} a_{n}(t) \sin \left(\lambda_{n} z\right) \\
& \bar{P}_{2}(z, t)=\sum_{n=0}^{\infty} b_{n}(t) \sin \left(\lambda_{n} z\right)
\end{aligned}
$$

where

$$
\lambda_{n}=\frac{(2 n+1) \pi}{2 h_{0}}
$$

Substitution of these general solutions in (74) and (75) yields

$$
\left[\begin{array}{ll}
a_{11} & a_{12} \\
a_{21} & a_{22}
\end{array}\right]\left[\begin{array}{l}
\frac{d a_{n}(t)}{d t} \\
\frac{d b_{n}(t)}{d t}
\end{array}\right]=\left[\begin{array}{l}
-e_{1} \lambda_{n}^{2} a_{n}(t) \\
-e_{2} \lambda_{n}^{2} b_{n}(t)
\end{array}\right]
$$

The initial values of $a_{n}(t)$ and $b_{n}(t)$ are obtained from (86) as

$$
a_{n}(0)=b_{n}(0)=\frac{2 P_{0}^{*}}{\lambda_{n} h_{0}}
$$

The application of the Laplace transformation to (90) yields

$$
\left[\begin{array}{cc}
s a_{11}+e_{1} \lambda_{n}^{2} & s a_{12} \\
s a_{21} & s a_{22}+e_{2} \lambda_{n}^{2}
\end{array}\right]\left[\begin{array}{l}
\overline{a_{n}}(s) \\
\bar{b}_{n}(s)
\end{array}\right]=\left[\begin{array}{ll}
a_{11} & a_{12} \\
a_{21} & a_{22}
\end{array}\right]\left[\begin{array}{l}
a_{n}(0) \\
b_{n}(0)
\end{array}\right]
$$

The solutions of the algebraic equations are

$$
\begin{aligned}
& \bar{a}_{n}(s)=\frac{a_{n}(0)\left[s \Delta+e_{2} \lambda_{n}^{2}\left(a_{11}+a_{12}\right)\right]}{s^{2} \Delta+s \lambda_{n}^{2}\left(a_{11} e_{2}+a_{22} e_{1}\right)+e_{1} e_{2} \lambda_{n}^{4}} \\
& \bar{b}_{n}(s)=\frac{b_{n}(0)\left[s \Delta+e_{1} \lambda_{n}^{2}\left(a_{21}+a_{22}\right)\right]}{s^{2} \Delta+s \lambda_{n}^{2}\left(a_{11} e_{2}+a_{22} e_{1}\right)+e_{1} e_{2} \lambda_{n}^{4}}
\end{aligned}
$$

where

$$
\Delta=a_{11} a_{22}-a_{21} a_{12}
$$

The inversion of (93) and (94) yields

$$
\begin{aligned}
& \overline{P_{1}}(z, t)=\sum_{n=0}^{\infty}\left(A_{n}^{1} e^{-r_{1} t}+A_{n}^{2} e^{-r_{2} t}\right) \sin \left(\lambda_{n} z\right) \\
& \overline{P_{2}}(z, t)=\sum_{n=0}^{\infty}\left(A_{n}^{3} e^{-r_{1} t}+A_{n}^{4} e^{-r_{2} t}\right) \sin \left(\lambda_{n} z\right)
\end{aligned}
$$

where

$$
\begin{gathered}
A_{n}^{1}=\frac{2 P_{0}^{*}}{\lambda_{n} h\left(r_{1}-r_{2}\right)}\left[r_{1}-\frac{e_{2} \lambda_{n}^{2}}{\Delta}\left(a_{11}+a_{12}\right)\right] \\
A_{n}^{2}=\frac{2 P_{0}^{*}}{\lambda_{n} h\left(r_{1}-r_{2}\right)}\left[-r_{2}+\frac{e_{2} \lambda_{n}^{2}}{\Delta}\left(a_{11}+a_{12}\right)\right] \\
A_{n}^{3}=\frac{2 P_{0}^{*}}{\lambda_{n} h\left(r_{1}-r_{2}\right)}\left[r_{1}-\frac{e_{1} \lambda_{n}^{2}}{\Delta}\left(a_{21}+a_{22}\right)\right] \\
A_{n}^{4}=\frac{2 P_{0}^{*}}{\lambda_{n} h\left(r_{1}-r_{2}\right)}\left[-r_{2}+\frac{e_{1} \lambda_{n}^{2}}{\Delta}\left(a_{21}+a_{22}\right)\right] \\
r_{1,2}=\frac{\lambda_{n}^{2}}{2 \Delta}\left(a_{11} e_{2}+a_{22} e_{1}\right) \pm \frac{1}{2} \sqrt{\frac{\lambda_{n}^{4}}{\Delta^{2}}\left(a_{11} e_{2}+a_{22} e_{1}\right)^{2}-\frac{4 e_{1} e_{2} \lambda_{n}^{4}}{\Delta}}
\end{gathered}
$$

The total settlement is calculated by

$$
\delta=-\int_{0}^{h_{0}} \frac{\partial \overline{w_{z}}}{\partial z} d z
$$

Substitution of (73), (96), and (97) in (103) yields

$$
\begin{aligned}
\delta= & \frac{P_{0} h}{K_{\mathrm{fr}}+4 G_{\mathrm{fr}} / 3}-\frac{1-K_{\mathrm{fr}} / K_{s}}{K_{\mathrm{fr}}+4 G_{\mathrm{fr}} / 3} \\
& \cdot\left\{\sum_{n=0}^{\infty} \frac{\left[S_{1} A_{n}^{1}+\left(1-S_{1}\right) A_{n}^{3}\right] e^{-r_{1} t}+\left[S_{1} A_{n}^{2}+\left(1-S_{1}\right) A_{n}^{4}\right] e^{-r_{2} t}}{\lambda_{n}}\right\}
\end{aligned}
$$

Note that the first term is the final settlement of the column when $t \rightarrow \infty$.

The parameters in Table 1 are used to obtain numerical results. Mechanical properties of the solid material are taken from Ishihara (1967) obtained for loose sand. Van Genuchten's (1980) closed form expressions for the capillary pressure-saturation relations are employed to obtain $d P_{\text {cap }} / d S_{1}$ in (31). Van Genuchten (1980) proposed that

$$
\frac{S_{2}-S_{r 2}}{S_{m 2}-S_{r 2}}=\left[1+\left(\frac{\alpha P_{\text {cap }}}{100}\right)^{n}\right]^{-m}
$$

where $P_{\text {cap }}=$ capillary pressure $\left(\mathrm{N} / \mathrm{m}^{2}\right) ; S_{2}=$ water saturation; $S_{m 2}=$ upper limit of water saturation in a two-phase medium; $S_{r 2}=$ irreducible water saturation; $m=1-1 / n$; and $\alpha$ and

TABLE 1. Model Parameters

\begin{tabular}{l|c|c}
\hline \multicolumn{1}{c|}{\begin{tabular}{c} 
Parameter \\
\multicolumn{1}{c|}{$(1)$}
\end{tabular}} & $\begin{array}{c}\text { Symbol } \\
(2)\end{array}$ & $\begin{array}{c}\text { Value } \\
(3)\end{array}$ \\
\hline Bulk modulus of solid matrix & $K_{\mathrm{fr}}$ & $0.0011 \mathrm{GPa}$ \\
Bulk of solid grains & $K_{s}$ & $37.04 \mathrm{GPa}$ \\
Shear modulus of solid matrix & $G_{\mathrm{fr}}$ & $0.007 \mathrm{GPa}$ \\
Intrinsic permeability & $K$ & $10^{-11} \mathrm{~m}^{2}$ \\
Volume fraction of solid phase & $\alpha_{s}$ & 0.7 \\
Bulk modulus of water & $K_{2}$ & $2.08 \mathrm{GPa}$ \\
Viscosity of water & $\mu_{2}$ & $10^{-3} \mathrm{~Pa} \cdot \mathrm{s}$ \\
Bulk modulus of oil & $K_{1}$ & $1.1 \mathrm{GPa}$ \\
Viscosity of oil & $\mu_{1}$ & $0.000647 \mathrm{~Pa} \cdot \mathrm{s}$ \\
Bulk modulus of air & $K_{1}$ & $0.14 \mathrm{MPa}$ \\
Viscosity of air & $\mu_{1}$ & $1.8 \times 10^{-6} \mathrm{~Pa} \cdot \mathrm{s}$ \\
External load & $P_{0}$ & $200,000 \mathrm{~Pa}$ \\
Thickness of column & $h_{0}$ & $10 \mathrm{~m}$ \\
\hline \hline
\end{tabular}


$n=$ material properties. For the relative permeabilities we use the following expression (Corapcioglu and Hossain 1990):

$$
k_{r 1}=\frac{\left[\left(1-S_{r 1}\right)-S_{2}\right]^{3}}{\left(1-S_{r 1}\right)^{2.5}} \text { and } k_{r 2}=\left(\frac{S_{2}-S_{r 2}}{1-S_{r 2}}\right)^{3}
$$

where $S_{r 1}=$ irreducible saturation of nonwetting phase. We will consider two cases as examples of practical problems. The first example is a porous column of an oil reservoir saturated by oil and water. The second is a column of unsaturated soil, i.e., air and water phase occupying the pore space.

\section{CASE 1: CONSOLIDATION OF POROUS COLUMN SATURATED BY OIL AND WATER PHASES}

We first analyze consolidation of a porous medium saturated by water and oil, characterized by material parameters of benzene obtained from Clark's Tables ("Clark's Tables" 1971). Parameters of (105) and (106) are obtained as $\alpha=0.025, n=$ $10, S_{r 2}=0.08$, and $S_{r 1}=0.10$ after curve matching the typical capillary pressure-saturation curve given by Wilson et al. (1990). The variation of $d P_{\mathrm{cap}} / d S_{1}$ with $S_{1}$, obtained from (105), is used to relate the pore pressure of the wetting phase to the pore pressure of the nonwetting phase by (31). As seen in Fig. 1, the dimensionless pore water pressure distribution along the column is dependent on water saturation. As expected, for larger water saturation values, excess water pressures are significantly higher. The effect of water saturation, $S_{2}$, is even more dramatic with increasing time. For example, at $t=10 \mathrm{~s}$, middepth $(z=5 \mathrm{~m})$ dimensionless excess pore pressure $\left(P_{2} / P_{0}^{*}\right)$ increases by $1 \%$ from 0.98 to 0.99 as the water saturation increases from $S_{2}=0.2$ to 0.5 . However, at a later time $(t=300 \mathrm{~s})$, the middepth excess pore pressures increase by 45 and $200 \%$ as $S_{2}$ increases from 0.2 to 0.3 and 0.5 , respectively. This reflects a substantial effect of oil presence as the medium consolidates. Then, change in oil saturation, $S_{1}$ can be calculated from (31). As shown in Fig. 2, at all times the decrease in oil saturation, $S_{1}$, along the column is relatively small. The increase in water saturation, $S_{2}$, is also equally small. Change in $\left(1-\alpha_{s}\right)$ (porosity) is calculated from (38). For parameters given in Table 1 , we observe the variation in porosity, as shown in Fig. 3. In $300 \mathrm{~s}$, we observe a change in porosity from 0.3 to 0.2975 at the lower boundary. An inspection of (50) indicates that when the upper limit of the

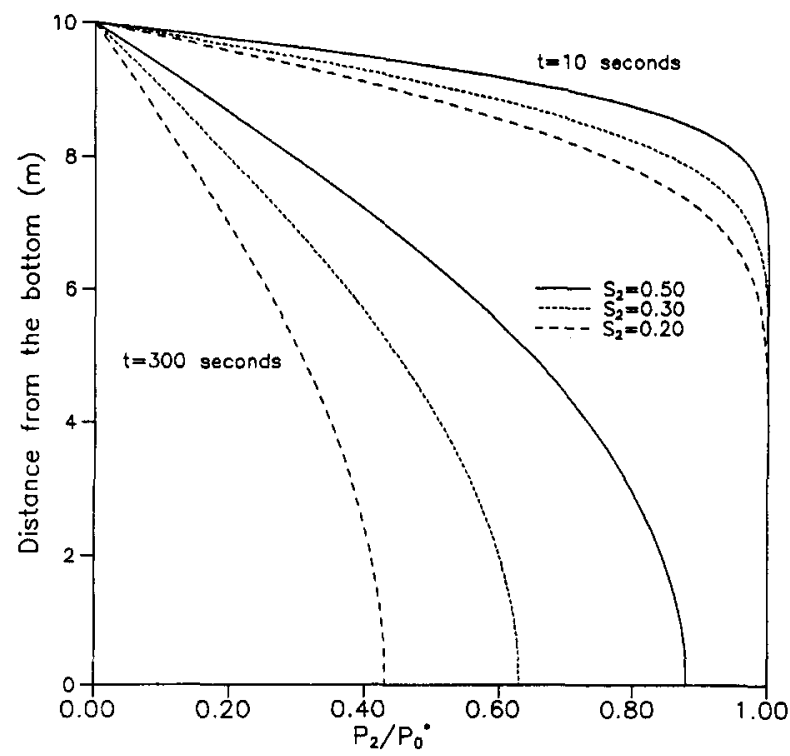

FIG. 1. Dimensionless Excess Pore Pressure Variation with Depth at $t=10$ and 300 s for Column Saturated by Oil-Water Phases

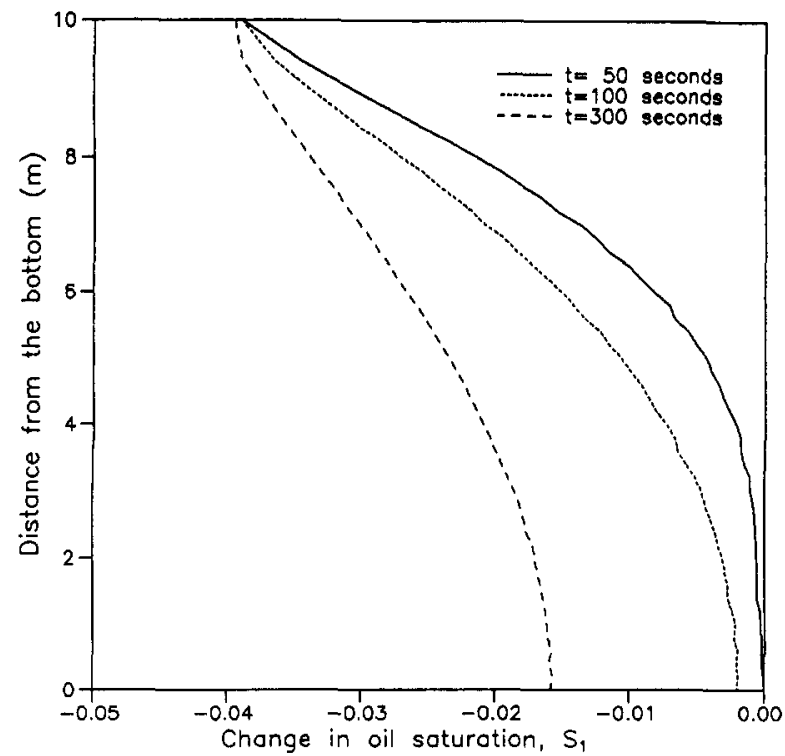

FIG. 2. Variation of Change in Oil Saturation with Depth for Water Saturation $S_{2}=\mathbf{0 . 3 0}$

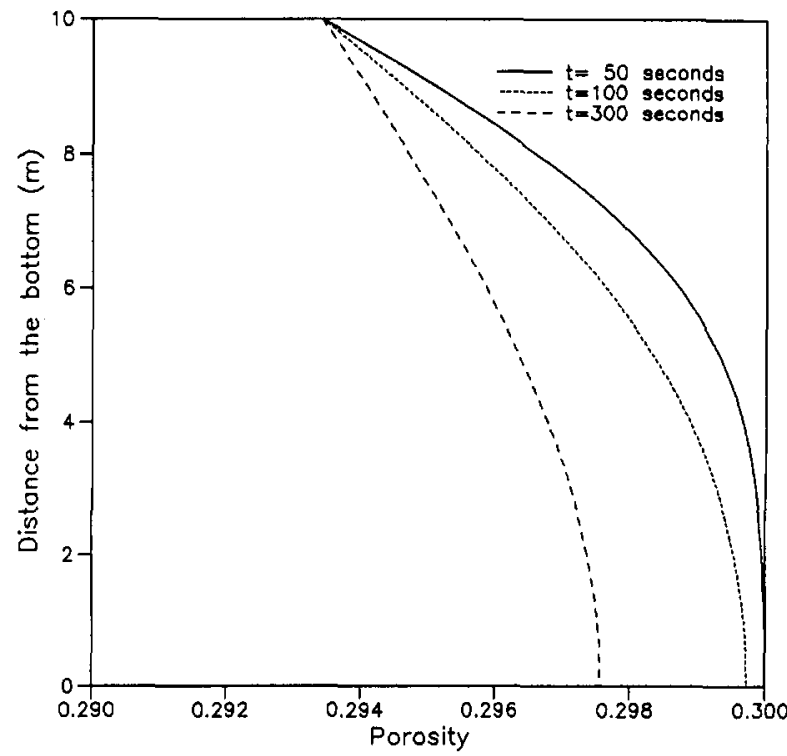

FIG. 3. Variation of Porosity with Depth for Water Saturation $s_{2}=0.30$

frame bulk modulus is employed, i.e., $K_{\mathrm{fr}}=\alpha_{s} K_{s}$, the change in porosity is zero.

\section{CASE 2: CONSOLIDATION OF POROUS COLUMN SATURATED BY AIR AND WATER PHASES}

In the second case, the column is saturated by air and water phases. Material properties are given in Table 1 ("Clark's Tables" 1971). Parameters of (105) and (106) are obtained as $\alpha=0.04, n=5, S_{r 2}=0.10$, and $S_{r 1}=0.05$. Fig. 4 illustrates the total settlement of a column fully saturated by water and of a column containing $5 \%$ air saturation. As shown in Fig. 4, although the final settlement and settlement at early times are not affected by the air presence, there is up to a $5 \%$ increase in settlement due to 5\% air saturation. As seen in Fig. 5, the dimensionless excess pore pressure distribution is affected by the air presence. For example, at $t=10 \mathrm{~s}$, middepth dimensionless excess pore pressure decreases by $1 \%$ from 0.99 to 0.98 as the water saturation decreases from full saturation, $S_{2}=1.00$ to $S_{2}=0.95$. At a later time, $t=300 \mathrm{~s}$, the middepth dimensionless excess pore pressure decreases by $15 \%$ as water 


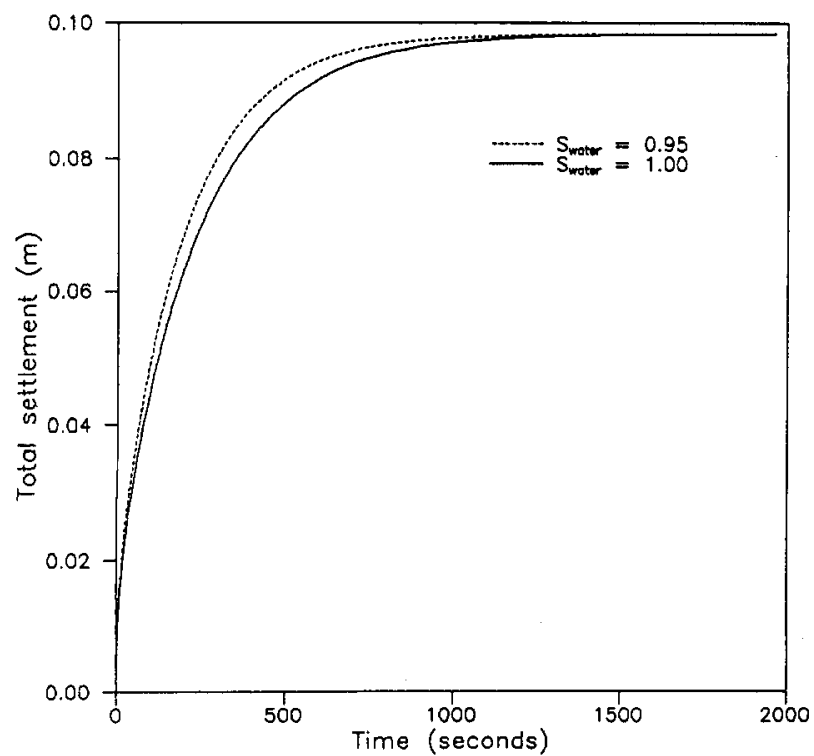

FIG. 4. Effect of Air Presence on Temporal Variation of Total Settlement

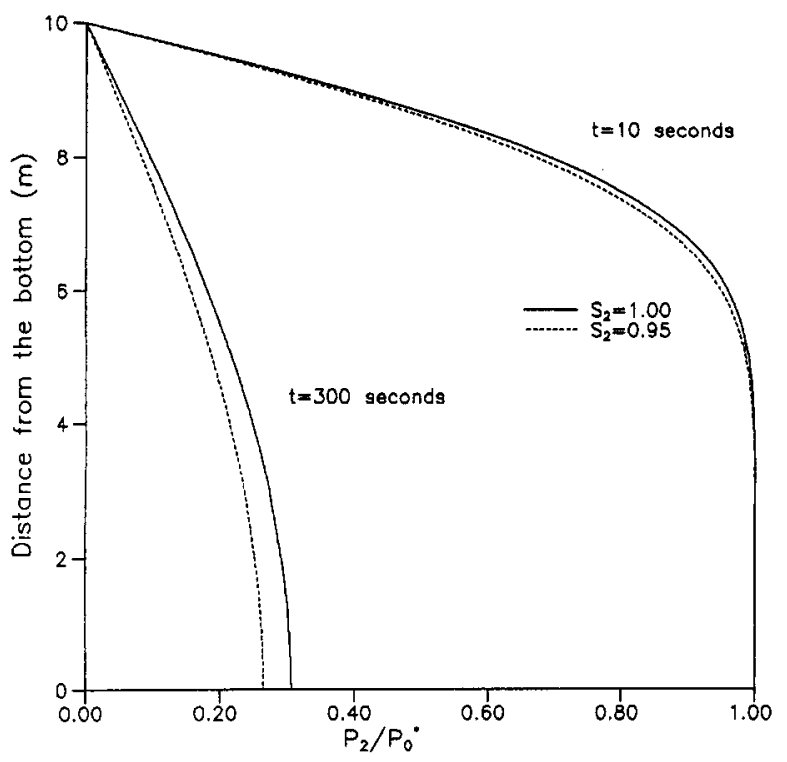

FIG. 5. Effect of Air Presence on Variation of Excess Pore Pressure with Depth at $t=10$ and $300 \mathrm{~s}$

saturation decreases by $5 \%$. This demonstrates the effect of air presence on the total settlement and pore water pressure.

\section{CONCLUSIONS}

We obtained macroscopic equations governing the consolidation of porous media saturated by two immiscible fluids by volume averaging the microscopic constitutive relations, and mass and momentum balance equations of respective phases. Momentum transfer terms are expressed in terms of intrinsic and relative permeabilities assuming the validity of Darcy's law. In one dimension, the governing equations reduce to two coupled diffusion equations in terms of the pore pressures of the fluid phases. An analytical solution is obtained for a column with a fixed impervious base and a free drainage surface. Results were presented for cases of practical interest, i.e., columns saturated by oil-water and air-water phases. It has been shown that the presence of a second fluid phase affects pore water pressure and total settlement. For example, it has been noted that in a column saturated by oil and water phases, the middepth excess pore pressures increase by 45 and $200 \%$ as the water saturation increases from 0.2 to 0.3 and 0.5 , respectively, although the change in porosity is from 0.3 to 0.2975 at the lower boundary. In an identical column saturated by air and water phases, the middepth dimensionless excess pore pressure decreases by $15 \%$ as the water saturation decreases by $5 \%$.

\section{APPENDIX. REFERENCES}

Adkins, J. E. (1963). "Nonlinear diffusion-I. diffusion and flow of mixtures of fluids." Trans., Royal Society London, 255, 607-634.

Anderson, T. B., and Jackson, R. (1967). "A fluid mechanical description of fluidized beds." I\&EC Fundamentals, 6, 527-539.

Bear, J. (1972). Dynamics of fluids in porous media. American Elsevier, New York, N.Y.

Bear, J., Corapcioglu, M. Y., and Balakrishna, J. (1984). "Modeling of centrifugal filtration in unsaturated deformable porous media." $A d v$. Water Resour., 7(4), 150-167.

Biot, M. A. (1941). "General theory of three-dimensional consolidation." J. Appl. Phys., 12(Feb.), 155-164.

Biot, M. A. (1956). "The theory of deformation of a porous viscoelastic anisotropic solid." J. Appl. Phys., 27(5), 459-467.

Biot, M. A., and Willis, D. G. (1957). "The elastic coefficients of the theory of consolidation." J. Appl. Mech., 24(Dec.), 594-601.

Bishop, A. W. (1959). "The principle of effective stress." Teknik Ukeblad, 39, 859-863.

Bishop, A. W., and Blight, G. E. (1963). "Some aspects of effective stress in saturated and partly saturated soils." Geotechnique, 13, 177-197.

Bowen, R. M. (1982). "Compressible porous media models by the use of the theory of mixtures." Int. J. Engrg. Sci., 20(6), 697-735.

Brutsaert, W., and El-Kadi, A. I. (1984). "The relative importance of compressibility and partial saturation in unconfined groundwater flow." Water Res. Resour., 20(3), 400-408.

"Clark's Tables." (1971). Science Data Base. Oliver and Boyd, Edinburgh, U.K.

Coleman, J. D. (1962), "Stress-strain relations for partly saturated soils." Geotechnique, 12, 348-350.

Corapcioglu, M. Y., and Bear, J. (1983). "A mathematical model for regional land subsidence due to pumping - 3 . integrated equations for a phreatic aquifer." Water Resour. Res., 19(4), 895-908.

Corapcioglu, M. Y., and Hossain, M. A. (1990). "Ground-water contamination by high density immiscible hydrocarbon slugs in gravity-driven gravel aquifers." Ground Water, 28(3), 403-412.

de la Cruz, V., and Spanos, T. J. T. (1985). "Seismic wave propagation in a porous medium." Geophysics, 50, 1556-1565.

de la Cruz, V., and Spanos, T. J. T. (1989). "Thermomechanical coupling during seismic wave propagation in a porous medium." J. Geophys. Res., 94, 637-642.

Fredlund, D. G., and Hasan, J. U. (1979). "One-dimensional consolidation theory: unsaturated soils." Can. Geotech. J., 16(3), 521-531.

Fredlund, D. G., and Morgenstern, N. R. (1976). "Constitutive relations for volume change in unsaturated soils." Can. Geotech. J., 13(3), $261-276$.

Fredlund, D. G., and Morgenstern, N. R. (1977). "Stress state variables for unsaturated soils." J. Geotech. Engrg., 103(5), 447-466.

Geraminegad, M., and Saxena, S. K. (1986). "A coupled thermoelastic model for saturated-unsaturated porous media." Geotechnique, 36, $539-550$.

Ishihara, K. (1967). "Propagation of compressional waves in a saturated soil." Proc., Int. Symp. Wave Propagation and Dyn. Properties of Earth Mat., Univ. of New Mexico Press, Albuquerque, N.M., 451 467.

Jennings, J. E. B., and Burland, J. B. (1962). "Limitations to the use of effective stresses in partly saturated soils." Geotechnique, 12, 125144.

Kohgo, Y., Nakano, M., and Miyazaki, T. (1993). "Theoretical aspects of constitutive modelling for unsaturated soils." Soils and Founds., $33(4), 49-63$.

Lloret, A., and Alonso, E. E. (1980). "Consolidation of unsaturated soils including swelling and collapse behavior." Geotechnique, 30, 449477.

Marle, C. M. (1967). "Ecoulements monophasiques en milleu poreux." Rev. Inst. Francais du Petrole, 22, 1471-1509.

Narasimhan, T. N., and Witherspoon, P. A. (1977). "Numerical model for saturated-unsaturated flow in deformable porous media-1. theory." Water Resour. Res., 13(3), 657-664.

Narasimhan, T. N., and Witherspoon, P. A. (1978a). "Numerical model for saturated-unsaturated flow in deformable porous media-2, the algorithm." Water Resour. Res., 14(2), 255-261. 
Narasimhan, T. N., and Witherspoon, P. A. (1978b). "Numerical model for saturated-unsaturated flow in deformable porous media-3. applications." Water Resour. Res., 14(6), 1017-1034.

Nur, A., and Byeerle, J. D. (1971). "An exact effective stress law for elastic deformation of rock with fluids." J. Geophys. Res., 74, 64146419.

Pride, S. R., Gangi, A. F., and Morgan, F. D. (1992). "Deriving the equations of motion for isotropic media." J. Acoust. Soc. Am., 88(Dec.), 3278-3290.

Scott, P. H., and Rose, W. (1953). "An explanation of the Yuster effect." J. Petr. Technol., 5, 19-20.

Skempton, A. W. (1960). "Effective stress in soils, concrete and rock." Pore Pressure and Suction in Soils, Butterworths, London, 1-13.

Slattery, J. C. (1967). "Flow of viscoelastic fluids through porous media." AlChE J., 13, 1066-1071.

Slattery, J. C. (1968). "Multiphase viscoelastic fluids through porous media." AIChE J., 14, 50-56.
Slattery, J. C. (1981). Momentum, energy and mass transfer in continua. Krieger, New York, N.Y.

Terzaghi, K. (1923). "Die berechnung der durchlaessigkeitsziffer des tones aus dem verlauf der hydrodynamicschen spannungserscheinungen." Sitzbericht (AbtIla). Akademia der Wissenschaften, Vienna, Austria, 132 (in German).

Whitaker, S. (1967). "Diffusion and dispersion in porous media." $A I C h E$ J., 13, 420-427.

Wilson, J. L., Conrad, S. H., Mason, W. R., Peplinski, W., and Hagon, E. (1990). "Laboratory investigation of residual liquid organics from spills, leaks and disposal of hazardous wastes in groundwater." U.S. Envir. Protection Agency Rep. No. EPA/600/6-90/004, Ada, Okla.

Van Genuchten, M. T. (1980). "A closed form equation for predicting the hydraulic conductivity of unsaturated soils." Soil, Sci. Soc, Am. J., $44,892-898$.

Yuster, S. T. (1953). "Theoretical considerations of multiphase flow in idealized capillary system." Proc., Third World Petr. Congr., The Hauge, The Netherlands, 2, 436-445. 